\title{
COVID-19: Companion Animals Help People Cope during Government-Imposed Social Isolation
}

\author{
Elizabeth Johnson | ORCID: 0000-0002-3914-6600 \\ Department of Anthropology, University of Nevada, Las Vegas \\ Shelly Volsche | ORCID: 0000-0002-0549-1318 \\ Department of Anthropology, Boise State University, Idaho \\ shellyvolsche@boisestate.edu
}

\begin{abstract}
This research explores the impact of government-imposed social isolation orders on homes with companion animals. Data were collected April through May 2020, the onset of the COVID-19 pandemic. A survey of 234 Americans observing social isolation orders included demographic questions; questions about relationships to other persons and companion animals within the home; and Likert-scale questions designed to probe the complexities of these relationships and their influences on perceived stress and isolation. We hypothesized that the presence of companion animals helps to mitigate stressors related to observing social isolation orders, with those living alone experiencing more benefit and homes with children experiencing less. The results suggest that the presence of companion animals alleviates stress and isolation by providing attachment figures and activities on which to focus one's energy. These results support that companion animals are increasingly viewed as members of one's family and provide social support during stressful life events.
\end{abstract}

\section{Keywords}

companion animals - human-nonhuman animal relations - social support - social isolation - COVID-19 


\section{Introduction}

Research demonstrates that human-nonhuman animal interactions can promote health by reducing stress, increasing overall well-being, and improving physical measures (i.e., heart rate, blood pressure, cortisol) via what is known as the "pet effect" (Barker, Barker, McCain, \& Schubert, 2016; Miller et al., 2009; Nagasawa et al., 2015). Companion animal guardians report higher self-esteem, more positive moods, more ambition, greater life satisfaction, and lower levels of loneliness (El-Alayli, Lystad, Webb, Hollingsworth, \& Ciolli, 2006), and are more satisfied with their lives than non-guardians (Bao \& Schreer, 2016).

Though the authors note it takes time for guardians to experience positive physiological benefits like reduced blood pressure or weight loss, Headey and Grabka (2011) suggest companion animals help to support daily exercise, reducing cortisol and improving the immune system response. Additionally, Uvnäs-Moberg (1998) suggests that reductions of blood pressure and cortisol levels result from this increased activity.

However, the evidence of the benefits of human-nonhuman animal interactions remain mixed (see Chur-Hansen, 2010; Herzog, 2011; Lass-Hennemann, Peyk, Streb, Holz, \& Michael, 2014; Peacock, Chur-Hansen, \& Winefield, 2012). While the literature documents some value of companion animals in mitigating self-reports of stress, it continues to be inconclusive given the many variables associated with guardianship, physiological and psychological experiences of stress, and the wide range of depth and quality of attachment regarding companion animals. Researchers attempt to address these methodological barriers (e.g., Saunders, Parast, Babey, \& Miles, 2017); others establish the potential negative impacts of companion animal guardianship, including a lack of compliance with one's own healthcare directives (Hodgson, Darling, \& Kim, 2015).

As we observed the notable increase in social media sharing of companion animal-related content when social isolation orders rolled through the United States in March and April of 2020, we sought to explore the impact of companion animals within various household structures. Many people are working from home or continuing to navigate employment in essential businesses. Engaging in social media via one's companion animal may be an outlet and welcome distraction, and it also serves to alleviate stressors such as children homeschooling, loss of employment, fear of infection, and feelings of isolation. It is important to understand how individuals manage this conflict, as evidence suggests social isolation and loneliness are linked to negative cardiovascular and mental health outcomes (Leigh-Hunt, Bagguley, Bash, Turner, Turnbull, Valtorta, \& Caan, 2017). 
The early days of the COVID-19 pandemic, with its subsequent governmentimposed social isolation orders, provided a unique opportunity to study the potential benefits and challenges of the pet effect in situ. Serpell and Paul (2002) provide historical evidence that human-companion animal relationships are emotional in nature and rely on familiarity and closeness. Many people provide companion animals with membership to human social groups (Midgley, 1983; Serpell, 1986). In exchange, companion animals may play a central role in an individual's life as authentic members of one's family unit, providing social and emotional support (Meehan, Massavelli, \& Pachana, 2017). Companion animals fulfill roles such as friends for young children in the home (Cassels, White, Gee, \& Hughes, 2017), replacement of children for some adults (Laurent-Simpson, 2017; Volsche, 2018), and an extension of one's self (Ramirez, 2006; Veevers, 1985).

This study explores the questions, "Are companion animals helping mitigate stress on other household relationships during shelter-in-place orders, and if so, how?" and "What roles do companion animals play within various household structures when families are under duress?" We hypothesized that companion animals act to relieve isolation for those living alone and mitigate the stress of interactions for those living with other adults or children. We anticipated this may occur by increasing a sense of social support via a positive attachment figure; providing an acceptable means to disengage from other family members; increasing one's physical activity; and providing a perceived mediator during intrapersonal conflict in the home.

\section{Materials and Methods}

\section{Participants}

We created an online survey using Qualtrics. During the consent process, all participants confirmed that they were at least 18 years old, living in the United States, and living with at least one companion animal. At the beginning of the survey, two questions served to further confirm or exclude individuals who did not live with at least one companion animal and/or were not currently observing government-imposed isolation orders in their area. Based on $\mathrm{G}^{*}$ Power software (Faul, Erdfelder, Lang, \& Buchner, 2007), approximately 255 participants were required to detect a moderate to large effect size. As such, our recruitment target was 300 respondents, anticipating a portion of collected data would not be viable for analysis.

Recruitment took place via the investigators' social media accounts, using snowball sampling and sharing/retweeting. Respondents clicked on 
a bit.ly short URL which directed them to Qualtrics to complete the survey. Participants accessed the survey anonymously, indicated informed consent prior to beginning the survey, and received no compensation. Exclusion criteria include those who do not have companion animals, are under the age of 18 , and are not participating in government-imposed isolation orders (e.g., essential workers). Due to the potentially sensitive nature of the topic, participants were also advised to reach out to a local mental healthcare professional if they became upset answering the survey.

\section{Survey Design}

There are key terms used in the survey that warranted definition in order to provide consistency. In the United States, many federal, state, and local agencies use the phrase "shelter in place" to refer to government-imposed social isolation orders. In the survey, we defined the term for our participants as "working from home; engaging in school from home; avoiding social gatherings; using drive-thru or delivery; avoiding discretionary travel; etc." This definition is based upon the White House's "3o Days to Slow the Spread (Coronavirus.gov)."

We defined the term "household" as all persons living in the home, including "spouse/partner, your child, your mother, roommate's child, etc." For the Likert questions about household relationships, we defined "family" as "household relationships; family or individuals currently living with you." This was done in addition to asking about household structures (i.e., number of adults, number of children). Finally, we used the term "pet" in our survey to refer to companion animals and remain consistent with United States cultural norms.

The survey consisted of demographic questions (i.e., age group, gender, education, racial/ethnic background), reporting household/family structure (live alone, roommate, significant other, long-distance partnership, family; as well as number of adults, number of children), and documentation of the presence of companion animals (i.e., species, years owned, pet's age). Likert-scale questions designed to probe respondents' perceptions of these relationships were developed with the goal of creating a scale that could be used to ask about both family and companion animals, resulting in a set of parallel questions. There were assorted open-ended questions and opportunities for respondents to elaborate on "yes/no" answers to questions such as "Has your relationship with any members of your household or pets changed after shelter in place?"

After the collection of demographic information, the survey was separated into two stages: "Questions about Relationships in the Household" (Relationships) and "Questions about Pets in the Household" (Pets). In our Relationships section, participants were asked to answer questions about their household, number of members currently living in their home, responsibilities 
related to these members, and their children's schooling. We also asked for relationship details about each member within the household and if participants felt that COVID-19 had strained any of these relationships. We provided open-ended options for additional information.

In our Pets section, participants answered questions about the number and species of companion animals in the home, where the companion animals sleep, what words are used to identify their relationships with their companion animals (parent, guardian, owner, friend, other), how they refer to their companion animals among others, and where their companion animals spend most of their time. We then asked participants to think about the companion animal that they spent the most time with during shelter in place, in order to prime their responses to the Pets version of the Likert-scale questions.

We developed 25 Likert-scale statements to probe the roles of companion animals and family in the home, as well as feelings of isolation, coping, and wellbeing. These statements were based upon Williams's $(1997,2001)$ four states (belonging, control, self-esteem, and meaningful existence) and the Lexington Attachment to Pets Scale (LAPS) (Johnson, Garrity, \& Stallones, 1992). These two instruments have been validated and overlap significantly in the phrasing and content of questions. Due to the timing of our study and the uncertainty of how long social isolation orders would remain in place, we did not test the validation of the combined and revised scales before launch.

The Likert scales included questions regarding how the respondent's relationships with their family and their companion animals are being impacted by observing shelter in place. (See Table 1 for a full list of these statements.) The prompt for all questions began, "Since observing the shelter in place order ..." and continued with 25 statements regarding the family or the companion animal. All statements were presented in the same order in the Relationships and Pets sections of the survey, with the appropriate rewording. For example, "My family helps me escape the stresses of shelter in place" was included on the Relationships portion of the survey, and the corresponding statement, "My pet helps me escape the stresses of shelter in place" was included in the Pets section.

This example allows us to capture whether this effect was stronger for the companion animal or the family. Additionally, "I spend more time exercising or socializing with my pet without my family" and "I spend more time exercising or socializing with my family without my pet," emphasized the comparison between family members and companion animals. We chose a forced choice, 4-point Likert scale (strongly agree, agree, disagree, strongly disagree) to eliminate ambiguity given the potentially sensitive nature of the questions. 
TABLE 1 Full list of Likert scale questions for relationships* section

Since observing the shelter in place order ...

I feel more "disconnected" from my family.

I feel rejected, ignored, or excluded by my family.

Loving my family helps me stay healthy.

I feel my family likes to interact with me more than before.

I am sharing more posts about my family on social media than before.

My family helps me escape the stresses of shelter in place.

I feel better about myself when around my family.

I feel liked by my family.

My family makes me feel happy.

I feel that everything I do with my family is an effort.

I enjoy the increased time spent with my family.

I feel invisible or meaningless to my family.

I feel more important or useful to my family than before.

My family helps me feel less anxious.

Most of my activities involve my family during shelter in place.

I tell more stories from my family's perspective on social media.

I feel like my family is an extension of me.

I feel my family values me more than before.

I feel I can influence the actions of others in my family.

I feel that my family takes time away from my pet since shelter in place.

I need time alone with my family, away from my pet.

My relationships with my family are much easier to maintain than my relationships with my pet since shelter in place.

I am more concerned about supplies for my family members than for my pet.

I spend more time exercising or socializing with my family without my pet.

I spend more time exercising alone than before shelter in place.

* The same list was used for relationships with the companion animal. The words "family" and "pet" were reversed in the second representation of the scale.

4-point Likert scale from " $1=$ Strongly Agree" to " 4 = Strongly Disagree"

Open-ended questions were used to understand nuance in the respondents' answers and lived experiences while observing shelter in place. These questions included, "Has your relationship with any members of your household or pets changed after shelter in place?" and "What coping strategies did you use during shelter in place?" We also asked "yes/no" questions such as, "Are 
you fostering an animal during shelter in place?" and "Do you feel that your pet helped you or is helping you cope with shelter in place?" with space and requests for participants to provide further explanation.

\section{Analysis}

Upon closing the survey, an initial raw file was exported to Excel from Qualtrics. After cleaning the data, we imported the file to SPSS V26. Statistical tests included Principal Components Analysis on the Likert scales, Mann-Whitney on the resulting scales, and $\chi$-squared tests to compare groups on "yes/no" questions such as "Do you feel your pet helped you or is helping you cope with shelter in place?" We present the findings of our analysis in Results.

\section{Results}

\section{Participants}

A total of 313 responses were collected via Qualtrics. We removed individuals who did not answer affirmatively to the consent question $(n=3)$, did not complete the survey according to Qualtrics's algorithm $(n=67)$, or were the result of duplicate IP addresses with a short (five minutes or less) response duration $(n=9)$. Our final sample $(n=234)$ identified as female $(n=194)$, male $(n=$ 27 ), gender non-conforming (GNC) $(n=5)$, queer $(n=3)$, trans $\mathrm{MtF}$ (male to female) $(n=2)$, and other (specifically stated non-binary) $(n=2)$. Additionally, one individual did not respond to the gender question. There were respondents from all age categories ranging from " $18-24$ " years to " $75^{+}$" years.

Herzog (2007) noted the challenge of obtaining diverse participants, particularly for surveys related to companion animals and/or attachment. The demographics of our respondents reflect this challenge. As is so common, our respondents are primarily female $(194,82.9 \%)$, heterosexual (181, 73.1\%), and White $(205,87.6 \%)$. Additional biases in our sample involve the rate of highly educated respondents (Bachelors $=82,35.0 \%$; Graduate or Professional $=112$, $47.9 \%)$; reported incomes of middle class $(\$ 39,476-82,000=68,29.1 \%)$ and upper middle class $(\$ 84,201-160,725=74,31.6 \%)$; and being married or living with a domestic partner for more than one year (95, 40.6\%). Likewise, most of our respondents reported having a house with a yard (170, 72.6\%). See Table 2 for a complete breakdown of the sample demographics. 
TABLE 2 Respondent demographics

\begin{tabular}{|c|c|c|c|}
\hline & $n(\%)$ & & $n(\%)$ \\
\hline Gender: & & Age: & \\
\hline Female & $194(82.9)$ & $18-24$ & $14(6.0)$ \\
\hline Male & $27(11.5)$ & $25-35$ & $71(30.3)$ \\
\hline GNC & $5(2.1)$ & $36-46$ & $53(22.6)$ \\
\hline Queer & $3(1.3)$ & $46-6 o$ & $54(23.1)$ \\
\hline Trans MtF & $2(0.9)$ & $61-75$ & $38(16.2)$ \\
\hline Other & $2(0.9)$ & $75^{+}$ & $2(0.9)$ \\
\hline Did not answer & $1(0.4)$ & Did not answer & $2(0.9)$ \\
\hline \multicolumn{2}{|l|}{ Ethnic/Racial Background: } & \multicolumn{2}{|l|}{ Sexual Orientation: } \\
\hline White & $205(87.6)$ & Heterosexual & $171(73.1)$ \\
\hline Hispanic or Latino/a & $15(6.4)$ & Bisexual & $36(15 \cdot 4)$ \\
\hline Asian or Asian American & $5(2.1)$ & Homosexual & $18(7 \cdot 7)$ \\
\hline Native American/ & $2(0.9)$ & Asexual & $4(1.7)$ \\
\hline Alaskan Native & & Other & $3(1.3)$ \\
\hline Other & $4(1.7)$ & Did not answer & $2(0.9)$ \\
\hline Did not answer & $3(1.3)$ & & \\
\hline Education: & & Income: & \\
\hline HS diploma or equiv. & $5(2.1)$ & $\$ 0-\$ 9,700$ & $3(1.3)$ \\
\hline Some college, no degree & $23(9.8)$ & $\$ 9,701-\$ 39,475$ & $39(16.7)$ \\
\hline Associate & $11(4 \cdot 7)$ & $\$ 39,476-\$ 84,200$ & $68(29.1)$ \\
\hline Bachelor & $82(35 \cdot 0)$ & $\$ 84,201-\$ 160,725$ & $74(31.6)$ \\
\hline Graduate or Professional & $112(47 \cdot 9)$ & $\$ 160,726-204,100$ & $22(9.4)$ \\
\hline \multirow[t]{3}{*}{ Did not answer } & $1(0.4)$ & $\$ 204,101-\$ 306,75^{\circ}$ & $13(5.6)$ \\
\hline & & $\$ 306,75^{1+}$ & $11(4 \cdot 7)$ \\
\hline & & Did not answer & $4(1.7)$ \\
\hline Household Structure:* & & Home Type: & \\
\hline Live alone & $45(19.2)$ & House with yard & $170(72.6)$ \\
\hline Roommate & $15(6.4)$ & House without yard & $8(3.4)$ \\
\hline Significant Other & $90(38.5)$ & Apartment/condo & $5^{1}(21.8)$ \\
\hline Family & $84(35.9)$ & Other & $5(2.1)$ \\
\hline
\end{tabular}

* A fifth category for household structure, "long-distance partnership," was collapsed into the category "significant other," as only two respondents chose this option.

GNC = gender nonconforming; HS = high school; equiv. = equivalent. 


\section{Household Structure}

We intended to compare homes with and without children present, but there were inconsistencies responding to the question, "Enter the number of children (persons age 17 or younger) currently living in your home." Several individuals who reported living alone also reported having a child living in their home. We asked this question immediately after "Enter the number of adults (persons age 18 or older) currently living in your home," with the first option being "1 (self)."

We suspect inattention, fatigue, or stress may have prompted some respondents to read the questions as identical. As such, rather than separating cases by presence or absence of children, we chose to use the proxy of "household type" resulting from the responses to the question, "Which of the following best describes your household." The options were "live alone," "roommate," "significant other," "long distance partnership," and "family." Only two respondents chose "long distance partnership," and that category was collapsed into "significant other." Ultimately, 45 (19.2\%) respondents identified as living alone, $15(6.4 \%)$ had roommates, 90 (38.5\%) lived with significant others, and $84(35.9 \%)$ lived with families. We further collapsed all categories beside "Family" into the group "No Children" to serve as a proxy for the presence of children (family) and absence of children (other groups, especially living alone and significant other) in the home. We continued with our analysis using these two proxy groups- "Family" and "No Children."

\section{Analysis of Likert Scale Questions}

We completed a Principal Components Analysis of the Likert-scale questions for each section (Relationships and Pets, respectively). For the Relationships section (probing relationships with human household members), all statements loaded strongly into one factor. We then attempted a PCA using a Varimax rotation, and the phenomenon remained, with all statements loading at 0.900 or higher.

After completing a PCA with Varimax rotation for the Pets section (probing relationships with companion animals), a difference emerged. The rotation converged in six iterations, and we compared the item loadings. Four of the original 25 items either failed to load or loaded equally to more than one scale. This left us with 21 items that loaded to one of three scales at o.69o or higher, with loading scores ranging from 0.691 ("I am sharing more posts about my pet on social media than before") to 0.935 ("My pet makes me feel happy"). A complete list of the factors, their included items, and loading scores are presented in Table 3 . 
TABLE 3 Items by scale with loading scores

Scale 1: Social Isolation and Companion Animals

I feel more "disconnected" from my pet.

I feel invisible or meaningless to my pet.

I feel my pet likes to interact with me more than before.

I feel my pet values me more than before.

I feel rejected, ignored, or excluded by my pet.

I feel liked by my pet.

My pet helps me feel less anxious.

I tell more stories from my pet's perspective on social media.

I feel that everything I do with my pet is an effort.

Scale 2: Companion Animals as Attachment Figure

My pet makes me feel happy.

I enjoy the increased time spent with my pet.

Loving my pet helps me stay healthy.

I feel I can influence the actions of my pet.

I feel better about myself when around my pet.

I feel more important or useful to my pet than before.

I am sharing more posts about my pet on social media than before.

Scale 3: Companion Animals vs. Family

I spend more time exercising or socializing with my pet without my family.

I spend more time exercising alone than before shelter in place.

My relationships with my pet are much easier to maintain than my

relationships with my family since shelter in place.

I am more concerned about supplies for my pet than for my family members.

I feel that my pet takes time away from my family since shelter in place.
0.827

Loading

0.913

0.913

0.912

0.912

0.912

0.911

0.909

0.908

0.792

Loading

0.935

0.934

0.839

0.766

0.695

0.692

0.691

Loading

0.901

0.818

0.800

0.763

Extraction Method: Principal Component Analysis.

Rotation Method: Varimax with Kaiser (rotation converged in 6 iterations).

Scale 1, Social Isolation and Companion Animals, includes statements related to perceptions of acceptance and alleviation of loneliness. This includes the statement "I tell more stories from my pet's perspective on social media," suggesting that companion animals help individuals connect with human others online when observing social-isolation orders. Other statements include "I feel my pet likes to interact with me more than before" and "My pet helps me feel 
less anxious." Scale 1 achieved an excellent Cronbach's alpha of o.993, with a total of nine items.

Scale 2, Companion Animals as Attachment Figure, represents the value of connection and spending time with companion animals. This scale includes statements such as "I enjoy the increased time spent with my pet" and "I feel more important or useful to my pet than before." Scale 2 also includes the statement "I am sharing more posts about my pet on social media than before." At first glance, it seems counterintuitive that this loads differently than the statement about sharing posts from the companion animal's perspective. However, one suggests seeking to alleviate social isolation via the companion animal as an extension of one's self, while the other seeks to connect via stories of a beloved attachment in one's life. We discuss this distinction further in our Discussion. Scale 2 achieved an excellent Cronbach's alpha of 0.959 , with a total of seven items.

Scale 3, Companion Animals vs. Family, includes statements regarding time or financial investment tradeoffs between human family members and companion animals. Here statements such as "I spend more time exercising or socializing with my pet without my family" and "I am more concerned about supplies for my pet than for my family members" probe one's sense of security with others in their home. The statement "I feel my pet takes time away from my family since shelter in place" also loaded to this scale. Scale 3 achieved an excellent Cronbach's alpha of o.939, with a total of five items.

\section{Comparing "Family" and "No Children"}

To compare the groups "Family" and "No Children" on the three scales, we completed a Mann-Whitney $U$ test. Our collapsed proxy groups consisted of $n=84$ for "Family" and $n=15$ o for "No Children." Because our data were not sufficiently normalized, we used a Probability of Superiority $(P S)$ test to assess the effect size of our results. Our Likert scales were measured from " $1=$ Strongly Agree" to "4 = Strongly Disagree," so lower scores suggest agreement. Table 4 provides the descriptions of our sample across all three scales.

When comparing Scale 1, Social Isolation and Companion Animals, there was no statistically significant difference between "Family" and "No Children" ( $U=$ $6146.5^{\circ}, p=0.755, P S=0.512$ ). Individuals in the group "No Children" were more likely to agree than "Family" on Scale 2, Companion Animals as Attachment Figure $(U=5323.00, p=0.048, P S=0.578)$, and Scale 3 , Companion Animals vs. Family $(U=4790.00, p=0.002, P S=0.620)$, with small to medium effect sizes. This is likely reflective of the different role companion animals play in many homes without children, which we consider in the Discussion. 
TABLE 4 Sample descriptives by scale

\begin{tabular}{|c|c|c|c|c|c|c|c|c|c|c|c|c|}
\hline & \multirow[b]{2}{*}{$n$} & \multirow[b]{2}{*}{$M$} & \multicolumn{3}{|c|}{ No Children } & \multicolumn{7}{|c|}{ Family } \\
\hline & & & $M d n$ & $S D$ & $g_{1}$ & $n$ & $M$ & $M d n$ & $S D$ & $g_{1}$ & $p$ & PS \\
\hline Scale 1 & $15^{\circ}$ & 2.143 & 2.889 & 8.318 & -12.229 & 84 & 1.492 & 2.889 & 11.175 & -8.982 & 0.755 & 0.512 \\
\hline Scale 2 & $15^{\circ}$ & 0.825 & 1.714 & 8.372 & $-11.5^{85}$ & 84 & -0.051 & 1.714 & 12.025 & -7.401 & 0.048 & 0.578 \\
\hline Scale 3 & $15^{\circ}$ & -0.204 & 2.600 & 15.460 & -5.784 & 84 & 1.014 & 2.900 & 11.690 & -7.867 & 0.002 & 0.620 \\
\hline
\end{tabular}

$g_{1}$ is the estimated skewness of the sample.

\section{Companion Animals and Coping}

We asked participants two "yes/no" questions with open-ended requests for elaboration, and we completed a $\chi$-squared test on the responses "yes" and "no" to determine if there were any significant differences across household types. "Has your relationship with any members of your household or pets changed after shelter in place?" was the first question; it had no statistically significant differences, with the majority of respondents in each group answering "no" ("Family" = 63.1\%, "No Children" = 66.7\%).

Relatedly, and more relevant to our research question, $87.6 \%$ of the total respondents answered "yes" to the question, "Do you feel your pet helped you or is helping you cope with shelter in place?" ("Family" $=82.1 \%$, "No Children" $=90.7 \%)$. The slightly higher percentage of homes without children agreeing with this statement, along with the near significance of the $\chi$ - squared test $\left(\chi^{2}=3.603, p=.058\right)$ further supports the importance of companion animals in homes without children. This is significant on its own, as it supports the value of companion animals in these homes during this stressful event.

\section{Discussion}

According to the Kaiser Family Foundation, nearly half of Americans reported negative mental health impacts as a result of the COVID-19 pandemic, potentially exacerbating pre-existing conditions and drug abuse (Panchal et al., 2020). This contrasts with previous literature suggesting government-imposed social isolation does not adversely impact mental health (Daily \& Kaplan, 2014). The intention of the current study was to explore, in situ, the impact 
of companion animals on households while observing government-imposed social isolation during the CoviD-19 pandemic. Our findings indicate that companion animals provide increased social support and play the role of social actors that can lower mental and emotional stress, by proxy providing physical benefits during shelter in place due to CoviD-19. This effect appears to be particularly important in homes without children.

There was strong agreement between both groups on Scale 1 (Social Isolation and Companion Animals, $p=0.755$ ). Because family is a key ingroup for most people, McConnell, Lloyd, and Humphrey (2019) argued that viewing a companion animal as a family member allowed companion animals to enhance feelings of social support and improve wellbeing. Previous research suggests group cohesion further improves mental health outcomes when someone is experiencing social isolation (Dailey \& Keplan, 2014). Hence, an existing sense of cohesion with one's companion animal may enhance feelings of emotional support during times of social isolation and stress. This may be important for all members of the household, not only those completing the survey.

Members of the group "No Children" were more likely to agree with statements in Scales 2 and 3 (Companion Animals as Attachment Figure and Companion Animals vs. Family). Companion animals may act as confidants, alleviating ostracism felt by a spouse or roommate. Caring for the companion animal may also symbolize caring for oneself. Veevers (1985) argues that companion animals may serve as an extension of oneself or as a surrogate human actor. This would explain our respondents' increase in sharing posts about companion animals on social media, as well as the overall sense of attachment reported by this group compared to those who have children on whom to focus their energy.

Companion animals may also bring household members together by conversation or tasks involving them. For example, Cloutier and Peetz (2016) found that shared commitment to companion animals served to strengthen couple identity and relationship maintenance behaviors. For romantic partners, expressing one's needs by proxy of their companion animals may remove barriers to making requests of the other person's time and emotional energy.

For individuals living alone or with significant others, the companion animal may serve as an outlet for nurturing and distraction via the application of parenting strategies. This may explain why these individuals are more likely to be concerned with the procurement of supplies for their companion animals (as measured on Scale 3). Conversely, they may simply not need to focus on children and other family members for whom they must provide. Additionally, individuals that live alone were more likely to exercise with their companion 
animal, an example of how caring for a companion animal may improve one's self-care. Finally, while individuals who live alone were the least likely to view their companion animal as an extension of the self, this may be due to the parent-like nature of the relationship.

Overall, our findings suggest that companion animals are viewed as members of these families and are included in social-isolation activities. Whether alleviating social isolation for an individual living alone, serving as an extension of the self to make requests of a significant other's time and energy, or providing a source of shared goals for a family with children, companion animals play an important role in providing social support that can mitigate the impact of social isolation. The processes underlying how nonhuman animals can be included in people's most important ingroups shed light on the psychology underlying how group memberships affect perceptions of humanity and improve mental health outcomes during times of duress.

Inherent in all research, especially survey research, are limitations related to sampling. As noted in the Results, our sample shows many of the typical biases of companion animal survey work. Our respondents were mostly female, white, heterosexual, and highly educated, meaning this survey may not be easily generalizable to the broader American population. However, the biases toward higher levels of education and living in homes with yards are likely indicative of who can work from home and observe social-isolation orders in their areas. Additionally, there is almost certainly a self-sampling bias of individuals who include their companion animals as family members and were willing to make the time to answer an online survey during the pandemic. Despite these limitations, we feel this study is still an important window into this specific population and their perceptions of and value placed on companion animals in their homes. It also interesting to note the similarities and differences in these perceptions based upon household type (presence or absence of children).

Relatedly, it is worth noting that we did not ask the specific ages of children in the home, focusing instead on the arbitrary line of being under 18 years old. Given the self-sampling bias of those who were able and willing to make the time to answer our survey, it is probable that we did not capture many individuals who have younger children with the need for more direct care. We suspect parents of younger children may find companion animals to be more of an additional obligation than a social support or respite from responsibilities in the home, but more data are needed to confirm this hypothesis.

Finally, there are some limitations related to the survey itself and the timing of data curation. To reduce potential harm to respondents, the survey was not designed to require responses to all questions. As a result, many respondents who reported living alone did not answer the Likert-scale questions related to Relationships. This left a gap in our data which may explain the lack of factors 
present in that section of the survey. Additionally, during recruitment, numerous surveys circulated social media related to covid, sheltering in place, and companion animals (including relinquishment and adoption). As such, we cannot rule out the impact of survey fatigue on recruitment as well as performance in answering the questions. This is particularly noteworthy during a time when mental health was becoming a topic of discussion due to social isolation and a turn toward increased screen time for work, school, and the management of relationships outside the home.

Despite these limitations, we feel our study adds value to the literature and provides thoughts for future research. For example, time diaries could be used to document changes in time spent with family and companion animals as the American economy begins to recover and people begin to leave their homes again. Time diaries, additional surveys, and interviews may also determine if and how leisure activities change in response to public health recommendations and a reevaluation of relationships. One question that may be asked is, "Do Americans return to the hectic, consumptive lifestyle from before the pandemic? Or do they maintain some of the focus on family, including companion animals, that social isolation orders brought to light?"

Future researchers should consider if and how policies related to companion animal adoption, enrichment, and quality of life do or need to change with the return to working outside the home. There is concern as to whether the increase in companion animal adoptions, labeled by the media as "pandemic puppies," may be setting up American shelters for an increase in unwanted companion animals when time and finances no longer allow for the maintenance of close bonds or the potential investment in addressing behavior issues sometimes wrought by drastic changes in daily routines. There are already conversations in professional companion animal services (i.e., veterinary, training) in preparation for the outreach and education needed to improve outcomes for companion animals should changes prove overwhelming for guardians.

\section{Conclusion}

In conclusion, while our study is not the first to document the perceived benefits and value of companion animals in the home, it is among the first to do so in real time, as a major stress event was, almost certainly, influencing these relationships. As the meaning of family and kinship continues to expand and include nonhuman animals, scholarly work must keep up to understand the role companion animals play within the home. While our sample reported benefits from and attention to maintaining these relationships during trying times, we understand this is not always the case and not all companion 
animals are held in such high regard. However, this does not negate that, for some individuals, companion animals truly do improve one's outcomes and coping with stress and social isolation. For these individuals, whether living alone or in a full house, their companion animals are important, affectionate attachment figures who are clearly worth the investment.

\section{References}

Bao, K.J., \& Schreer, G. (2016). Pets and happiness: Examining the association between pet ownership and wellbeing. Anthrozoös, 29(2), 283-296. https://doi.org/10.1080/o 8927936.2016.1152721.

Barker, S.B., Barker, R.T., McCain, N.L., \& Schubert, C.M. (2016). A randomized crossover exploratory study of the effect of visiting therapy dogs on college student stress before final exams. Anthrozoös, 29(1), 35-46. https://doi.org/10.108o/o8927936.2015 .1069988 .

Cassels, M., White, N., Gee, N., \& Hughes, C. (2017). One of the family? Measuring young adolescents' relationships with pets and siblings. Journal of Applied Developmental Psychology, 49, 12-2o. https://doi.org/10.1016/j.appdev.2017.01.003.

Chur-Hansen, A. (2010). Grief and bereavement issues and the loss of a companion animal: People living with a companion animal, owners of livestock, and animal support workers. Clinical Psychologist, 14(1), 14-21. https://doi.org/ $10.1080 / 13284201003662800$.

Cloutier, A., \& Peetz, J. (2016). Relationships' best friend: Link between pet ownership, empathy, and romantic relationship outcomes. Anthrozoös, 29(3), 395-408. https:// doi.org/10.108o/o8927936.2016.1181361.

Dailey, S.F., \& Kaplan, D. (2014). Shelter-in-place and mental health: An analogue study of well-being and distress. Journal of Emergency Management, 12(2), 121-131. http:// doi.org/10.5055/jem.2014.0166.

El-Alayli, A., Lystad, A.L., Webb, S.R., Hollingsworth, S.L., \& Ciolli, J.L. (20o6). Reigning cats and dogs: A pet-enhancement bias and its link to pet attachment, pet-self similarity, self-enhancement, and well-being. Basic and Applied Social Psychology, 28(2), 131-143. https://doi.org/10.1207/s15324834basp28o2_3.

Faul, F., Erdfelder, E., Lang, A.-G., \& Buchner, A. (2007). G*Power 3: A flexible statistical power analysis program for the social, behavioral, and biomedical sciences. Behavior Research Methods, 39(2), 175-191. https://doi.org/10.3758/BFo3193146.

Headey, B., \& Grabka, M. (2011). Health correlates of pet ownership from national surveys. How animals affect us: Examining the influence of human-animal interaction on child development and human health, 153-162. https://doi.org/10.1037/12301-0o8.

Herzog, H. (2007). Gender differences in human-animal interactions: A review. Anthrozoös, 2o(1), 7-21. https://doi.org/10.2752/o89279307780216687. 
Herzog, H. (2011). The impact of pets on human health and psychological well-being: Fact, fiction, or hypothesis? Current Directions in Psychological Science, 20(4), 236239. https://doi.org/10.1177/og63721411415220.

Hodgson, K., Barton, L., Darling, M., Antao, V., Kim, F., \& Monavvari, A. (2015). Pets' impact on your patients' health: Leveraging benefits and mitigating risk. Journal of American Board of Family Medicine, 28(4), 526-534. https://doi.org/o.3122/ jabfm.2015.04.140254.

Johnson, T., Garrity, T., \& Stallones, L. (1992). Psychometric evaluation of the Lexington Attachment to Pets Scale (Laps). Anthrozoös, 5(3), 16o-175. https://doi .org/10.2752/o89279392787011395.

Lass-Hennemann, J., Peyk, P., Streb, M., Holz, E., \& Michael, T. (2014). Presence of a dog reduces subjective but not physiological stress responses to an analog trauma. Frontiers in Psychology, 5, 1010. https://doi.org/10.3389/fpsyg.2014.01010.

Laurent-Simpson, A. (2017). "They make me not wanna have a child": Effects of companion animals on fertility intentions of the childfree. Sociological Inquiry, 87(4), 586-6o7. https://doi.org/10.1111/soin.12163.

Leigh-Hunt, N., Bagguley, D., Bash, K., Turner, V., Turnbull, S., Valtorta, N., \& Caan, W. (2017). An overview of systematic reviews on the public health consequences of social isolation and loneliness. Public Health, 152, 157-171. https://doi.org/10.1016/j .puhe.2017.07.035.

McConnell, A., \& Lloyd, E.P., \& Humphrey, B. (2019). We are family: Viewing pets as family members improves wellbeing. Anthrozoös, 32(4), 456-47o. https://doi.org/10 $.1080 / 08927936.2019 .1621516$.

Meehan, M., Massavelli, B., \& Pachana, N. (2017). Using attachment theory and social support theory to examine and measure pets as sources of social support and attachment figures. Anthrozoös, 3o(2), 273-289. https://doi.org/10.108o/o8927936 .2017.1311050.

Midgley, M. (1984). Animals and why they matter. Athens, GA: University of Georgia Press.

Miller, S., Kennedy, C., DeVoe, D., Hickey, M., Nelson, T., \& Kogan, L. (2009). An examination of changes in oxytocin levels in men and women before and after interaction with a bonded dog. Anthrozoös, 22(1), 31-42. https://doi.org/ 10.2752/175303708X390455.

Nagasawa, M., Mitsui, S., En, S., Ohtani, N., Ohta, M., Sakuma, Y., Onaka, T., Mogi, K., \& Kikusui, T. (2015). Oxytocin-gaze positive loop and the coevolution of human-dog bonds. Science, 348(6232), 333-336. https://doi.org/10.1126/science.1261022.

Panchal, N., Kamal, R., Orgera, K., Cox, C., Garfield, R., Hamel, L., Muñana, C., \& Chidambram, P. (2020, April 21). The implications of COVID-19 for mental health and substance use. Kaiser Family Foundation, Retrieved July 1, 2020: https://www.kff.org/coronavirus-covid-19/issue-brief/the-implications-of-covid -19-for-mental-health-and-substance-use/. 
Peacock, J., Chur-Hansen, A., \& Winefield, H. (2012). Mental health implications of human attachment to companion animals. Journal of Clinical Psychology, 68(3), 292-303. https://doi.org/10.1002/jclp.20866.

Ramirez, M. (2006). "My dog's just like me": Dog ownership as a gender display. Symbolic Interaction, 29(3), 373-391. https://doi.org/10.1525/si.20o6.29.3.373.

Saunders, J., Parast, L., Babey, S., \& Miles, J. (2017). Exploring the differences between pet and non-pet owners: Implications for human-animal interaction research and policy. PLOS One, 12(6), eo179494. https://doi.org/10.1371/journal.pone.0179494.

Serpell, J. (1986). In the company of animals: A history of human-animal relationships. Cambridge, United Kingdom: Cambridge University Press.

Serpell, J., \& Paul, E. (2002). Pets and the development of positive attitudes to animals. In Animal \& human society, 165-182. New York, NY: Routledge.

Uvnäs-Moberg, K. (1998). Oxytocin may mediate the benefits of positive social interaction and emotions. Psychoneuroendocrinology, 23(8), 819-835. https://doi .org/10.1016/So3o64530(98)ooo56-o.

Veevers, J. (1985). The social meaning of pets. Marriage \& Family Review, 8(3-4), 11-30. http://doi:10.130o/Joo2vo8no3_3.

Volsche, S. (2018). Negotiated bonds: The practice of childfree pet parenting. Anthrozoös, 37(3), 367-377. https://doi.org/10.108o/o8927936.2018.1455470.

Williams, K.D. (1997). Social ostracism. In Aversive interpersonal behaviors, 133-170. https://doi.org/10.1007/978-1-4757-9354-3_7.

Williams, K.D. (2001). Ostracism: The power of silence. New York, NY: Guilford Press. 